Article

\title{
Enhanced Liver Targeting of Camptothecin via Conjugation with Deoxycholic Acid
}

\author{
Linxia Xiao ${ }^{1}$, Endian $\mathrm{Yu}^{2}$, Hanlin Yue ${ }^{2}$ and Qingyong $\mathrm{Li}^{1,2, *}$ \\ 1 Collaborative Innovation Center of Yangtze River Region Green Pharmaceuticals, Zhejiang University of \\ Technology, Hangzhou 310014, China; xiaolinxiaxlx@126.com \\ 2 College of Pharmaceutical Science, Zhejiang University of Technology, Hangzhou 310014, China; \\ yu19960216@126.com (E.Y.); yhl333666@126.com (H.Y.) \\ * Correspondence: liqy@zjut.edu.cn; Tel.: +86-571-88320984
}

Received: 16 January 2019; Accepted: 21 March 2019; Published: 26 March 2019

check for updates

\begin{abstract}
Camptothecin (CPT) shows potent anticancer activity through inhibition of topoisomerase I. However, its water insolubility and severe toxicity limit its clinical application. Coupling with bile acid moieties is a promising method for liver-targeted drug delivery, which takes advantage of the bile acid receptors on hepatocytes. In this study, we evaluated the potential liver targeting and stability of a deoxycholic acid-CPT conjugate (G2). The competitive inhibition of antitumor activity experiment based on bile acid transporters was performed using the MTT method. The effects of deoxycholic acid on uptake of G2 and CPT were assessed in 2D and 3D HepG2 cell models. The stability of G2 and CPT was evaluated in vitro (in simulated gastric fluid, simulated intestinal fluid, and fresh rat plasma). Finally, biodistribution of G2 and CPT was investigated in Kunming mice following oral administration. The results showed that deoxycholic acid pretreatment could significantly reduce the antitumor activity and cellular uptake of G2 in HepG2 cells, but had no distinct effects on CPT. Meanwhile, G2 exhibited better stability compared with CPT. More importantly, biodistribution study in mice demonstrated that the liver targeting index of G2 increased 1.67-fold than that of CPT. Overall, the study suggests that conjugation with deoxycholic acid is a feasible method to achieve liver targeting delivery of CPT.
\end{abstract}

Keywords: camptothecin; bile acid; liver targeting; antitumor activity; uptake; biodistribution

\section{Introduction}

CPT (Figure 1A), a natural monoterpene-quinoline alkaloid first isolated from extracts of the Chinese tree Camptotheca acuminata, exhibits anticancer activity through inhibition of topoisomerase I [1-4]. As a potent inhibitor of DNA topoisomerase I, CPT shows high activity against a broad spectrum of cancers, including breast, small-cell lung, ovarian, pancreas cancer, particularly hepatic and colon cancer [5-8]. Unfortunately, the clinical application of CPT has been hindered by its water insolubility, severe toxicity to normal tissues and poor stability of the lactone ring [9-11]. Considerable modifications have been made to overcome these disadvantages. Among the synthesized analogues, irinotecan and topotecan have been approved for clinical therapy of certain cancers, including colon cancer, ovarian cancer, lung cancer and so on [12]. However, toxicities to normal tissues of both drugs are severe because of the non-targeted effects, which limits their utilization [13]. Therefore, the targeted delivery of CPT may lower its accumulation in other tissues and reduce its side effects. 
A<smiles>CC[C@@]1(O)C(=O)OCc2c1cc1n(c2=O)Cc2cc3ccccc3nc2-1</smiles>

CPT
B

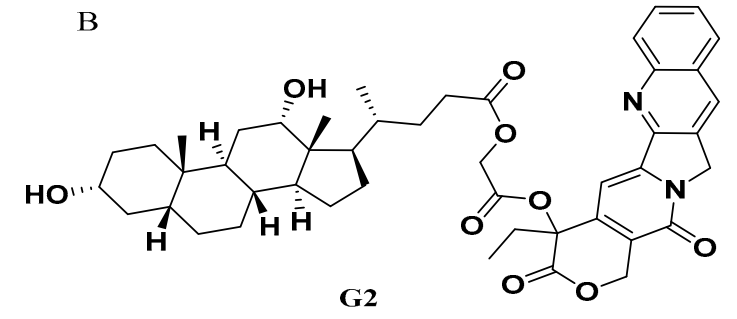

Figure 1. Chemical structures of CPT (A) and G2 (B).

The prodrug strategy, applying bile acids as the modifying moieties to target hepatocyte transporters and thus obtain hepatic-targeting delivery agents, has been suggested in previous studies [14-17]. This prodrug approach takes advantage of the highly specific interaction of bile acid ligands with $\mathrm{Na}^{+}$-dependent taurocholate co-transporting polypeptide (NTCP) receptors which are specifically and abundantly expressed in hepatocytes [18-20]. Furthermore, as a part of the enterohepatic circulation, happening 6-15 times per day, bile acids possess potent transport capacity [21]. Hence, by coupling bile acid moieties to CPT, the uptake of drug-loaded systems into hepatocytes could be enhanced with a high degree of selectivity and the toxicities of the obtained conjugates might be reduced.

Based on the above background, our laboratory synthesized a series of CPT-bile acid analogues in order to improve the tumor-targeting chemotherapeutic effects of CPT on liver cancer, thereby decreasing the toxicity of $\mathrm{CPT}$, and evaluated their antitumor activities [22,23]. Among these analogues, the compound G2 (Figure 1B) showed the highest anticancer activity in vitro and had a broad spectrum of anticancer activity [22]. G2 was synthesized by conjugating the CPT hydroxyl group at C20 position to the deoxycholic acid carboxyl group at C24 position via a linker. In order to evaluate whether G2 possesses the capacity to target the liver, competitive inhibition experiments were performed to investigate the effects of corresponding bile acid on the anticancer activity and cellular uptake of $\mathbf{G} 2$ in both 2D and 3D HepG2 cell model. Additionally, the in vitro stability of G2 and CPT was evaluated. Finally, the in vivo distribution of $\mathbf{G} 2$ was also examined in mice, in comparison with CPT.

\section{Results and Discussion}

\subsection{Effect of Deoxycholic Acid on Cytotoxicity of G2 and CPT}

In order to evaluate whether the deoxycholic acid-CPT conjugate G2 could be taken up by bile acid transporters, a competitive inhibition of antitumor activity experiment was performed.

As shown in Figure 2, pretreatment with deoxycholic acid markedly reduced the cytotoxicity of G2 against HepG2 cells. After pretreatment with $1 \mu \mathrm{M}$ deoxycholic acid for $0.5 \mathrm{~h}$, the cell viability of HepG2 exposured to G2 increased from $51.26 \%$ to $71.13 \%$. Meanwhile, no distinct effects of deoxycholic acid pretreatment on the cytotoxicity induced by CPT were revealed. Based on these results, we surmised that $\mathbf{G} 2$ could be taken up by bile acid transporters due to its conjugation with deoxycholic acid, which might increase intracellular accumulation of G2 in HepG2 cells. 


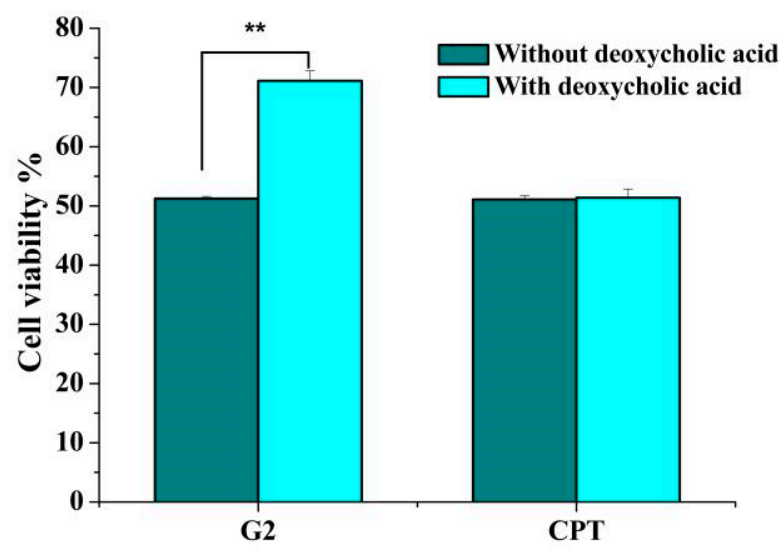

Figure 2. The effect of deoxycholic acid on cytotoxicity of G2 and CPT. After pretreatment with $1 \mu \mathrm{M}$ deoxycholic acid for $0.5 \mathrm{~h}$, HepG2 cells were incubated with $1 \mu \mathrm{M} \mathrm{G} 2$ or CPT for $48 \mathrm{~h}$ and the cell viability was detected. Results are presented as mean \pm SD of three independent measurements. ** $p<0.01$ compared with control group.

\subsection{Effects of Deoxycholic Acid on Uptake of G2 and CPT in 2D Cell Model}

In order to further confirm the hypothesis, the effects of deoxycholic acid on uptake of G2 and CPT were investigated. Pretreatment with deoxycholic acid significantly attenuated the uptake of G2 towards HepG2 cells in 2D cell model in a dose-dependent manner (Figure 3). The uptake of CPT towards HepG2 cells in 2D cell model showed no obvious change after pretreatment with deoxycholic acid. Meanwhile, without deoxycholic acid pretreatment, the uptake of $\mathbf{G} 2$ was 4.16-fold higher than that of CPT. Li et al. also discovered that cholic acid could reduce cellar uptake of cholic acid modified silybin towards HepG2 cells [17]. The inhibitory effect might be exerted via saturating NTCP expressed on HepG2. These results indicated that conjugation with deoxycholic acid changed the transport pathway of CPT and the uptake mediated by bile acid transporters played an important role in the cellular internalization of $\mathbf{G} 2$ in HepG2 cells.

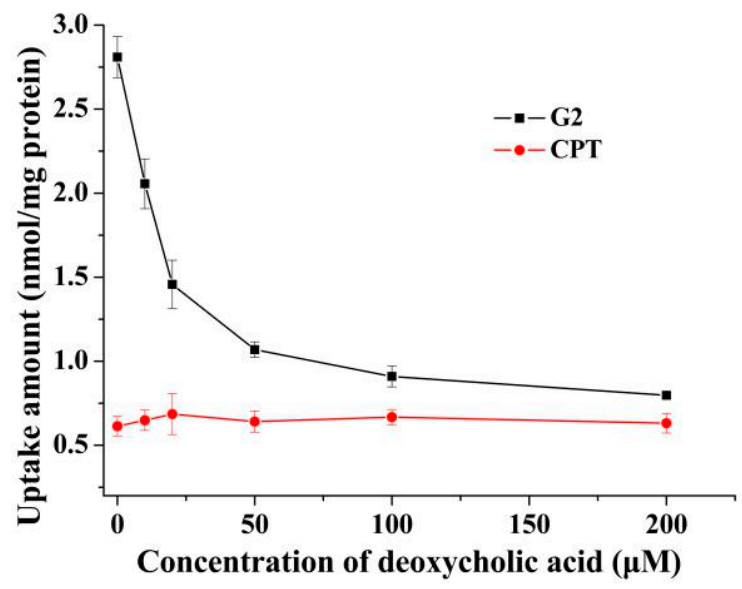

Figure 3. The effect of deoxycholic acid on uptake of G2 and CPT in HepG2 2D cell model. After pretreatment with different concentrations of deoxycholic acid for $0.5 \mathrm{~h}$, cells were incubated with $10 \mu \mathrm{M} \mathrm{G} 2$ or CPT for $2 \mathrm{~h}$ and the amount of uptake was detected. Results are presented as mean $\pm \mathrm{SD}$ of three independent measurements.

\subsection{Effects of Deoxycholic Acid on Uptake of G2 and CPT in 3D Cell Model}

Although 2D monolayer cell models have been used most frequently for in vitro investigations, they lack the extracellular matrix and 3D architecture of intact tumor tissue [24,25]. Therefore, 2D cell models can't sufficiently represent the in vivo tumors. In order to further understand the penetration 
of G2 and CPT into tumor tissue, a 3D HepG2 spheroid cell model was used to investigate the effect of deoxycholic acid on uptake of $\mathbf{G} 2$ and CPT, for its more similar to intact tumor [26]. As revealed in Figure 4, except for the decreased compound concentration of uptake, similar results to 2D cell model were obtained in 3D cell model. After pretreatment with $200 \mu \mathrm{M}$ deoxycholic acid, the cellar uptake of G2 in 3D cell model was decreased $66.4 \%$ compared to the group of deoxycholic acid-free. Whereas, there was no distinct effect of deoxycholic acid on cellar uptake of CPT. These results further demonstrated that $\mathbf{G} 2$ might be more exposed in the liver than CPT.

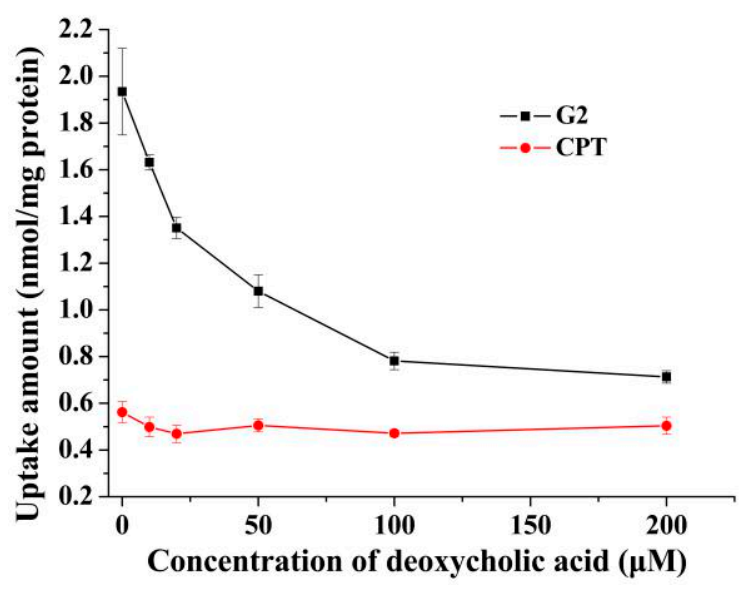

Figure 4. The effect of deoxycholic acid on uptake of G2 and CPT in HepG2 3D cell model. After pretreatment with different concentrations of deoxycholic acid for $0.5 \mathrm{~h}$, spheroids were incubated with $10 \mu \mathrm{M} \mathrm{G} 2$ or CPT for $2 \mathrm{~h}$ and the amount of uptake was detected. Results are presented as mean \pm SD of three independent measurements.

\subsection{Stability of $G 2$ and $C P T$}

Because the instability of the lactone ring is one of the reasons limiting the application of CPT, conjugation with deoxycholic acid at 20-position of CPT was developed to improve its stability and target the liver for its expression of bile acid transporters. Therefore, we measured the stability of G2 and CPT in simulated gastric fluid (SGF) and simulated intestinal fluid (SIF) as well as fresh rat plasma. As revealed in Figure 5, conjugate $\mathbf{G} 2$ effectively decreased hydrolysis compared with CPT in all of the three media, particularly in SGF and SIF. G2 was most stable in SIF. After $3 \mathrm{~h}$ incubation in SIF, G2 had no obvious degradation, whereas $16.1 \%$ of CPT were degraded. After $3 \mathrm{~h}$ incubation in SGF, the degraded rate of $\mathbf{G 2}(15.0 \%)$ was less than that of CPT (37.9\%).

In rat plasma, G2 was also more stable than CPT, with $42.3 \%$ and $24.4 \%$ remaining after $3 \mathrm{~h}$ incubation, respectively. These results indicated that conjugation with deoxycholic acid at 20-position of CPT increased the stability of lactone ring, which was consistent with previous reports $[27,28]$. 

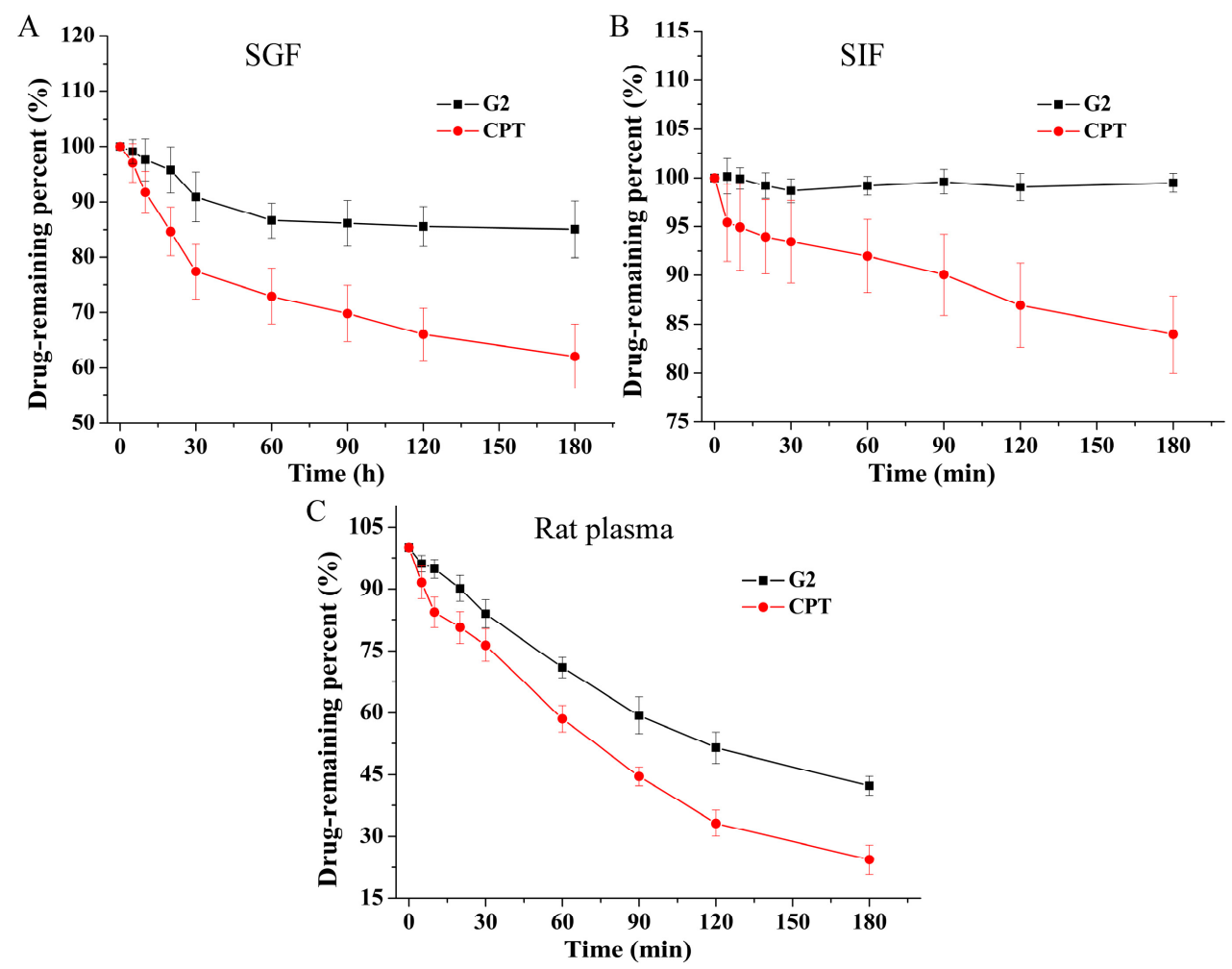

Figure 5. Stability of G2 and CPT was studied in (A) simulated gastric fluid (SGF), (B) simulated intestinal fluid (SIF) and (C) fresh rat plasma.

\subsection{Method Validation}

There was no significant endogenous interference for both the plasma and tissue samples (Figure 6). Calibration curves, correlation coefficients and linear ranges were summarized in Table S1 (Supplementary Materials). All correlation coefficients were greater than 0.99 , which indicated good linearity. For G2, the intra-day precision was no more than $8.90 \%$, and the accuracy was range from $90.67 \%$ to $108.24 \%$; the inter-day precision was no more than $6.91 \%$, and the accuracy was range from $93.06 \%$ to $104.49 \%$ (Table S2, Supplementary Materials). For CPT, the intra-day precision was no more than $8.11 \%$, and the accuracy was range from $95.22 \%$ to $105.54 \%$; the inter-day precision was no more than $6.08 \%$, and the accuracy was range from $94.73 \%$ to $106.18 \%$ (Table S2). Recovery in plasma and tissue homogenates were ranged from $82.40 \%$ to $91.48 \%$ and $81.66 \%$ to $93.06 \%$ for G2 and CPT, respectively (Table S3, Supplementary Materials). Furthermore, there was no significant matrix effect for the determination of G2 and CPT (Table S3). For the freeze-thaw, long-term and short-term stability investigations, the concentrations of G2 and CPT in plasma and tissue homogenates diverged no more than $\pm 15 \%$ from nominal values (Table S4, Supplementary Materials). 

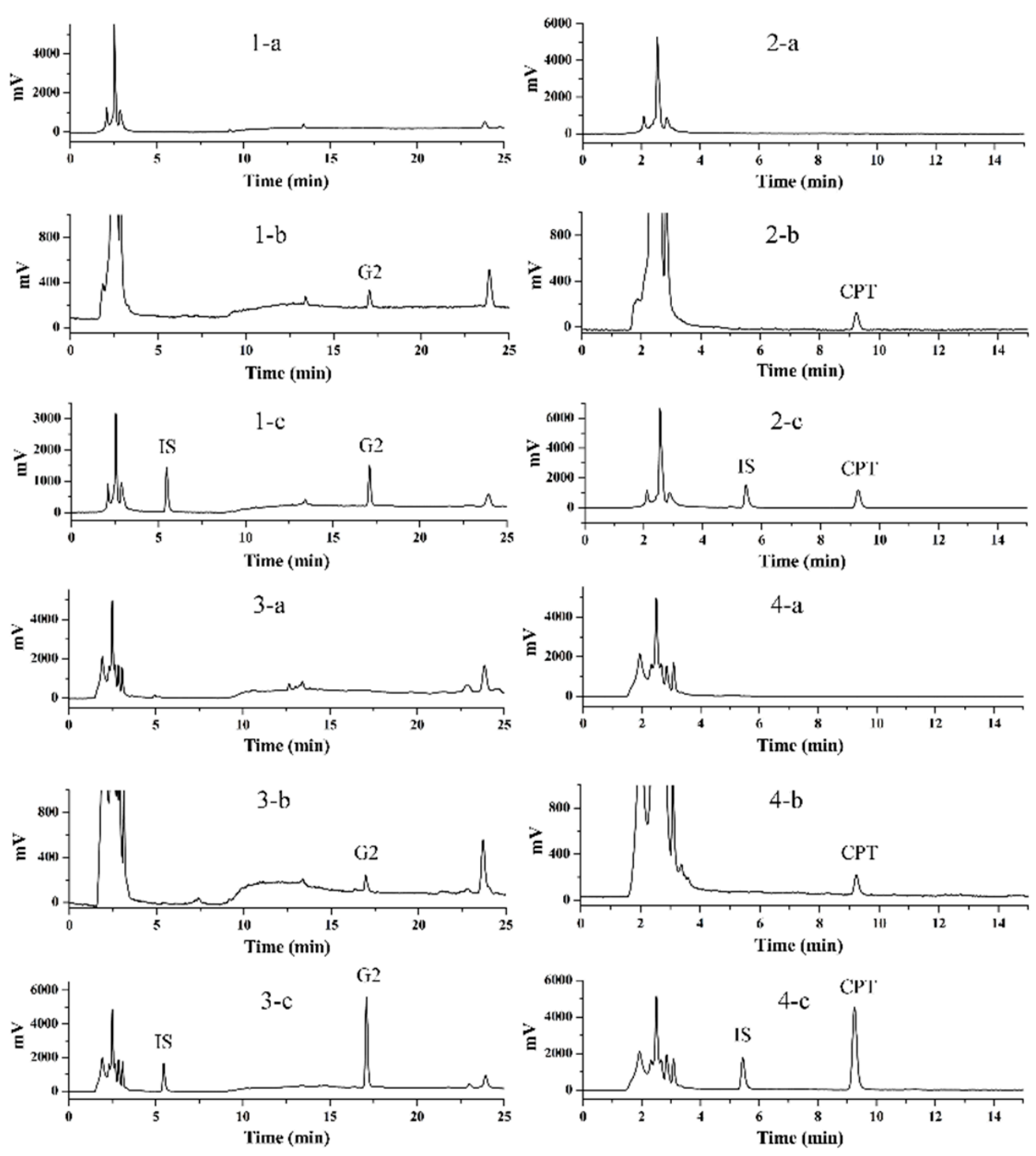

Figure 6. Chromatograms of plasma $(1,2)$ and liver homogenate $(3,4)$ samples: (a) blank sample; (b) blank sample spiked with $0.030 \mu \mathrm{M} \mathrm{G2}$ or CPT; (c) plasma or liver homogenate sample after oral administration $0.5 \mathrm{~h}$ and spiked with IS.

\subsection{Body Distribution}

Based on the results of the in vitro studies, a biodistribution of $\mathbf{G 2}$ and CPT was investigated in mice to evaluate the potential of liver targeting of deoxycholic acid conjugate G2 in vivo. After G2 or CPT intragastric administration, the concentrations of G2 or CPT were determined in the plasma and organs. The mean concentration-time curves of G2 and CPT in plasma and tissues were showed in Figure 7 and the main pharmacokinetic parameters obtained utilizing non-compartmental model were presented in Table 1. For CPT, the $\mathrm{AUC}_{0-\mathrm{t}}$ was highest in lung, but it was highest in liver for G2. This result indicated that conjugation with deoxycholic acid increased liver accumulation. In addition, compared with CPT, the AUC of deoxycholic acid conjugate G2 increased in all of the plasma and organs. Meanwhile, it increased most significantly in liver than other organs. After oral administration, the $\mathrm{AUC}_{0-\mathrm{t}}$ of $\mathrm{G} 2$ in liver was $16.04 \pm 2.78 \mathrm{nmol} \mathrm{h} / \mathrm{mL}$, which was 2.31-fold higher than that of CPT. And the LTI was 8.07 and 4.84 for G2 and CPT, respectively. It demonstrated that conjugation with deoxycholic acid increased liver targeting in vivo by 1.67-fold in comparison to CPT. This result was in accordance with previous report that conjugation with bile acid could increase liver targeting of ribavirin by 1.80 -fold [16]. 
A

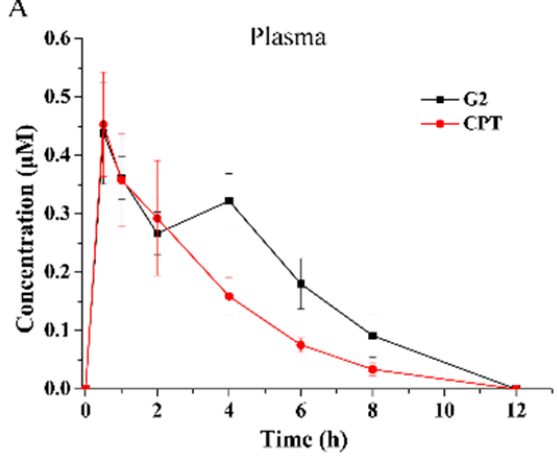

C
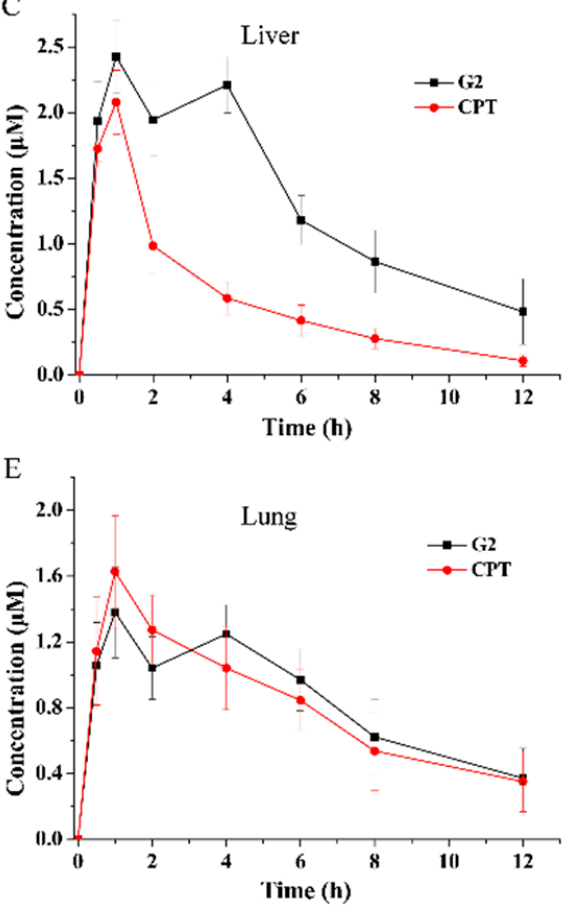

B

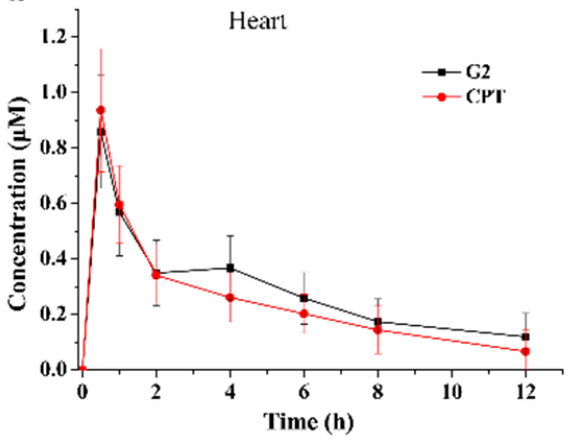

D

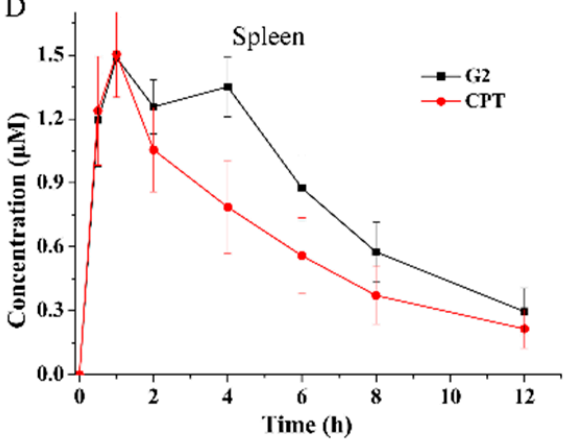

F

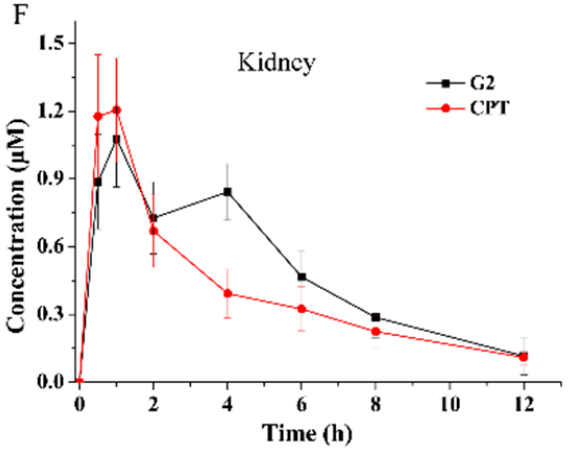

Figure 7. Total drug concentration-time profiles of $\mathrm{G} 2$ and CPT in plasma and organs after oral (30 mg/kg, calculated as CPT) administration: (A) plasma; (B) heart; (C) liver; (D) spleen; (E) lung; (F) kidney. Results are presented as mean $\pm \mathrm{SD}$ of five mice.

Table 1. Pharmacokinetic parameters of $\mathrm{G} 2$ and CPT after oral (30 mg/kg, calculated as CPT) administration (mean $\pm S D, n=5$ ).

\begin{tabular}{ccccccc}
\hline Analyte & Organ & $\mathbf{T}_{\max } \mathbf{( h )}$ & $\begin{array}{c}\mathbf{C}_{\mathbf{m a x}} \\
\mathbf{( n m o l} / \mathbf{m L})\end{array}$ & $\begin{array}{c}\mathbf{A U C}_{\mathbf{0 - t}} \\
(\mathbf{n m o l ~ h / m L )}\end{array}$ & $\begin{array}{c}\mathbf{A U C}_{\mathbf{0}-\boldsymbol{\infty}} \\
(\mathbf{n m o l ~ h} / \mathbf{m L})\end{array}$ & $\mathbf{M R T}_{\mathbf{0}-\boldsymbol{\infty}}(\mathbf{h})$ \\
\hline \multirow{6}{*}{$\mathbf{G} 2$} & Plasma & $0.600 \pm 0.200$ & $0.441 \pm 0.084$ & $1.987 \pm 0.319$ & $2.303 \pm 0.500$ & $4.355 \pm 0.645$ \\
& Heart & $0.600 \pm 0.200$ & $0.899 \pm 0.165$ & $3.362 \pm 1.065$ & $4.589 \pm 1.937$ & $7.799 \pm 3.102$ \\
& Liver & $1.000 \pm 0.000$ & $2.425 \pm 0.279$ & $16.043 \pm 2.783$ & $20.093 \pm 6.046$ & $7.194 \pm 2.298$ \\
& Spleen & $1.600 \pm 1.200$ & $1.526 \pm 0.140$ & $10.371 \pm 1.382$ & $12.112 \pm 2.056$ & $6.379 \pm 1.352$ \\
& Lung & $1.600 \pm 1.200$ & $1.386 \pm 0.269$ & $10.180 \pm 2.318$ & $12.889 \pm 4.211$ & $7.446 \pm 1.748$ \\
& Kidney & $1.800 \pm 1.166$ & $1.096 \pm 0.193$ & $6.055 \pm 1.197$ & $6.627 \pm 1.740$ & $5.049 \pm 1.003$ \\
\hline \multirow{6}{*}{ CPT } & Plasma & $0.500 \pm 0.000$ & $0.465 \pm 0.084$ & $1.556 \pm 0.260$ & $1.677 \pm 0.252$ & $3.224 \pm 0.578$ \\
& Heart & $0.600 \pm 0.200$ & $0.954 \pm 0.207$ & $2.860 \pm 0.983$ & $3.639 \pm 1.615$ & $6.350 \pm 2.712$ \\
& Liver & $1.000 \pm 0.000$ & $2.079 \pm 0.243$ & $6.954 \pm 1.319$ & $7.459 \pm 1.539$ & $4.261 \pm 0.380$ \\
& Spleen & $0.900 \pm 0.200$ & $1.526 \pm 0.195$ & $7.562 \pm 1.718$ & $9.292 \pm 2.527$ & $6.958 \pm 2.948$ \\
& Lung & $1.200 \pm 0.400$ & $1.641 \pm 0.325$ & $9.798 \pm 2.594$ & $12.431 \pm 4.429$ & $7.248 \pm 1.775$ \\
& Kidney & $0.800 \pm 0.245$ & $1.328 \pm 0.218$ & $4.827 \pm 1.045$ & $5.448 \pm 1.193$ & $5.322 \pm 0.460$ \\
\hline
\end{tabular}




\section{Materials and Methods}

\subsection{Materials}

The deoxycholic acid-CPT conjugate G2 (Figure 1B, CPT-20 (S)-O-acetate-deoxycholic acid) was synthesized and purified by our laboratory [22], to a purity beyond 99\%. The human hepatocellular carcinoma cell line HepG2 was purchased from the China Center for Type Culture Collection (Wuhan, China). Dulbecco's modified Eagle's medium (DMEM) was obtained from Gibco BRL (Grand Island, NY, USA). Dimethyl sulfoxide (DMSO) was purchased from Sigma-Aldrich (St. Louis, MO, USA). Fetal bovine serum (FBS) was obtained from Hyclone (Thermo Fisher Scientific, Logan, UT, USA). Phosphate-buffered saline (PBS), Hank's buffered salt solution (HBSS), penicillin and streptomycin were supplied by Genom (Hangzhou, China). Methyl thiazolyl tetrazolium (MTT) and trypsin were obtained from Solarbio (Beijing Solarbio Science \& Technology Co., Ltd., Beijing, China). All reagents for HPLC were of analytical grade.

\subsection{Cell Culture}

The human hepatocellular carcinoma cell line HepG2 were cultured in T-25 cm² cell culture flasks (Corning ${ }^{\circledR}$, Wujiang, China) with media consisting of DMEM supplemented with $10 \%(v / v)$ FBS, $100 \mathrm{U} / \mathrm{mL}$ penicillin, and $100 \mu \mathrm{g} / \mathrm{mL}$ streptomycin. Cells were maintained at $37^{\circ} \mathrm{C}$ with $5 \% \mathrm{CO}_{2}$ and $95 \%$ relative humidity. After $90 \%$ confluence, cells were digested by trypsin and passaged at a 1:3 split ratio.

\subsection{D Multicellular Tumor Spheroids Culture}

The liquid overlay method was employed for the culturing of HepG2 spheroids as described previously [29]. Cells reached logarithmic growth phase were digested by trypsin then centrifuged. The cell number was counted using a hemocytomete and cells were diluted in growth medium to a density of $1 \times 10^{4}$ cells $/ \mathrm{mL}$. Spheroids were seeded by pipetting $2 \mathrm{~mL}$ of the cell suspension into each well of a 6-well ultra-low attachment (ULA) plates (Corning ${ }^{\circledR}$, Wujiang, China) and incubated for 3 days.

\subsection{Competitive Inhibition Assays}

The effect of deoxycholic acid on anticancer activity of G2 against HepG2 cells was studied by MTT assay as described previously with slight modifications [30]. After reaching logarithmic growth phase, HepG2 cells were passaged and $100 \mu \mathrm{L}$ of the cell suspension was seeded into 96-well plates at a density of $5 \times 10^{4}$ cells $/ \mathrm{mL}$, then cells were cultured in an incubator (Thermo Fisher Scientific, Marietta, Ohio, USA) at $37^{\circ} \mathrm{C}$. After reached $80 \%$ confluence, cell monolayers were firstly incubated with or without $1 \mu \mathrm{M}$ deoxycholic acid for $0.5 \mathrm{~h}$. After pretreatment with deoxycholic acid, cells were washed with PBS buffer and $200 \mu \mathrm{L}$ of $1 \mu \mathrm{M}$ CPT or G2 was added. Next, cells were further incubated at $37^{\circ} \mathrm{C}$ for $48 \mathrm{~h}$. Then $20 \mu \mathrm{L}$ of MTT reagent $(5 \mathrm{mg} / \mathrm{mL}$ in PBS) was added and cells were incubated for another $4 \mathrm{~h}$ at $37^{\circ} \mathrm{C}$ to allow MTT to be metabolized into formazan. Finally, the media was tapped out and $150 \mu \mathrm{L}$ of DMSO was added to each well to dissolve the formazan crystals. After mixing, the absorbance was quantified at $490 \mathrm{~nm}$ using an ELISA Plate Reader (infinite M200 Pro, TECAN, Grodig, Salzburg, Austria).

\subsection{Uptake of G2 into 2D Cell Model}

Cellular uptake of G2 and CPT into 2D HepG2 cell model was performed as described previously with slight modifications [31]. After reaching logarithmic growth phase, HepG2 cells were passaged and $2 \mathrm{~mL}$ of the cell suspension was seeded into 6 -well plates at a density of $5 \times 10^{4}$ cells $/ \mathrm{mL}$. Then, cells were cultured in an incubator at $37{ }^{\circ} \mathrm{C}$. After reached $80 \%$ confluence, cell monolayers were pretreated with deoxycholic acid at different concentrations for $0.5 \mathrm{~h}$. Next, the inhibitor of deoxycholic 
acid was removed and cells were washed twice with Hank's balanced salt solution (HBSS). After $2 \mathrm{~h}$ incubation with $10 \mu \mathrm{M}$ G2 or CPT, cells were washed three times with HBSS and digested with $200 \mu \mathrm{L}$ of trypsin for 2 min to obtain single cell suspensions. Next, the cells were washed three times with cold HBSS and $250 \mu \mathrm{L}$ of HBSS was added to resuspend the pellets. Then, the cells were lysed by freeze-thaw three times and sonicated for $10 \mathrm{~min}$. After centrifugation, the supernatants were used for the measure of the compound and protein concentration. For determination of the compound concentration, $200 \mu \mathrm{L}$ of the supernatant was added to equal volume of acetonitrile to precipitate proteins, then the mixture was vortexed and mixed for $2 \mathrm{~min}$. After centrifugation, the supernatant was collected and filtered with a $0.22 \mu \mathrm{m}$ filter to acquire the sample for HPLC analysis. The compound concentration was calculated by the corresponding standard curve. The protein concentration of the supernatant was determined by the Bradford method based on a standard curve made by desired concentrations of bovine serum albumin. The cellular uptake was presented as the quantity of $\mathbf{G} 2$ or CPT associated with $1 \mathrm{mg}$ of cellular protein [32,33].

\subsection{Uptake of G2 into 3D Cell Model}

After HepG2 spheroids were prepared as 3.3, they were transferred into $4 \mathrm{~mL}$ centrifuge tube and washed twice with HBSS. Then, the spheroids were resuspended with deoxycholic acid at different concentrations and seeded into the 6-well ULA plates again. After being cultured in an incubator for $0.5 \mathrm{~h}$ at $37^{\circ} \mathrm{C}$, the spheroids were transferred into $4 \mathrm{~mL}$ centrifuge tube and washed twice with HBSS to remove the deoxycholic acid. Then, they were resuspended with $10 \mu \mathrm{M} \mathrm{G} 2$ or CPT and seeded into the 6-well ULA plates. After being cultured in an incubator for $2 \mathrm{~h}$ at $37^{\circ} \mathrm{C}$, the spheroids were transferred into $4 \mathrm{~mL}$ centrifuge tube and washed three times with HBSS. Then, the spheroids were digested with $200 \mu \mathrm{L}$ of trypsin for $5 \mathrm{~min}$ to obtain single cell suspensions and subsequent processing was the same as $2 \mathrm{D}$ cell model.

\subsection{Stability of $G 2$}

The stability of G2 and CPT was assessed in SGF, SIF and fresh rat plasma. SGF and SIF were prepared according to a previous report with slight modifications [34]. Briefly, 0.3\% (w/v) purified pepsin, $2 \mathrm{~g}$ of $\mathrm{NaCl}$ and $7 \mathrm{~mL}$ of $\mathrm{HCl}(12 \mathrm{M})$ were dissolved in $1000 \mathrm{~mL}$ of distilled water to obtain SGF (PH 1.2). To make SIF (PH 6.8), $6.8 \mathrm{~g}$ of $\mathrm{KH}_{2} \mathrm{PO}_{4}, 1.0 \%(w / v)$ purified pancreatin and $77 \mathrm{~mL}$ of $\mathrm{NaOH}(0.2 \mathrm{M})$ were dissolved in $1000 \mathrm{~mL}$ of distilled water. SGF, SIF and rat plasma containing $10 \mu \mathrm{M}$ $\mathrm{G} 2$ or $\mathrm{CPT}$ were incubated at $37^{\circ} \mathrm{C}$. At scheduled time points $(0,5,10,20,30,60,90,120,180 \mathrm{~min})$, $100 \mu \mathrm{L}$ samples were collected and reaction was quenched immediately by adding $400 \mu \mathrm{L}$ of ice cold acetonitrile. The mixture was votexed $2 \mathrm{~min}$ and centrifuged at $12000 \mathrm{rpm}$ for $10 \mathrm{~min}$. Then, the supernatant was filtered with a filter $(0.22 \mu \mathrm{m})$ and detected by HPLC.

\subsection{In Vivo Distribution of G2 in Mice}

For body distribution investigations, 80 male Kunming mice (weight, 18-22 g; age, 6-8 weeks) were used. The animal experiments in this study were performed under the guidance of the care and use of laboratory animals in Zhejiang University of Technology, Hangzhou, China, and conformed to the National Institutes of Health Guide for Care and Use of Laboratory Animals (Publication No. 85-23). After fasting overnight, mice were received a single dose of G2 or CPT $(30 \mathrm{mg} / \mathrm{kg}$, calculated as CPT) suspension by gavage. At desired time $(0.5,1,2,4,6,8,12 \mathrm{~h})$, animals were sacrificed. Their blood samples were collected by eyeball removal and immediately centrifuged to separate plasma. The organs (heart, liver, spleen, lung, kidney) were removed and weighed before homogenization. And the plasma and tissue homogenate samples were stored at $-80{ }^{\circ} \mathrm{C}$ until analysis.

\subsection{Calibration Curves and Quality Control Samples Preparation}

G2, CPT and 10-hydroxycamptothecin (IS) were dissolved in DMSO at $1 \mathrm{mM}$ to obtain the standard stock solutions. The standard working solutions $(0.3,0.6,1.2,3,6,12,24,48 \mu \mathrm{M}$ for G2 and 
CPT; $2 \mu \mathrm{M}$ for IS) were prepared by diluting the stock solutions with acetonitrile. The calibration standards $(0.015,0.03,0.06,0.15,0.3,0.6,1.2,2.4 \mu \mathrm{M})$ were prepared by spiking $5 \mu \mathrm{L}$ of standard working solutions into $95 \mu \mathrm{L}$ of blank plasma or tissue homogenate. The preparation of quality control samples $(0.03,0.3,1.2 \mu \mathrm{M})$ was same as the calibration standards. All solutions were stored at $4{ }^{\circ} \mathrm{C}$ until further use.

\subsection{Plasma and Tissue Homogenate Samples Processing}

For determination of the compound concentration, $200 \mu \mathrm{L}$ methanol and $100 \mu \mathrm{L}$ acetonitrile were added to $100 \mu \mathrm{L}$ plasma or tissue homogenate to precipitate protein, and $5 \mu \mathrm{L}$ IS $(2 \mu \mathrm{M})$ was also added. Mixtures were votexed for $2 \mathrm{~min}$ and centrifuged at $12,000 \mathrm{rpm}$ for $10 \mathrm{~min}$ at $4{ }^{\circ} \mathrm{C}$. Then, supernatant was transferred to a new tube and evaporated by an EZ-2 personal solvent evaporator (Genevac, Suffolk, UK). Finally, $50 \mu \mathrm{L}$ of $50 \%$ acetonitrile $(v / v)$ was added to re-dissolve the samples. After centrifuged at 13,000 rpm for $10 \mathrm{~min}$, the supernatant was used for HPLC analysis.

\subsection{HPLC Analysis}

Both G2 and CPT were measured by HPLC analysis, which was performed using a Shimadzu HPLC system (Shimadzu, Kyoto, Japan). To determine G2 and CPT concentration in both cell models, an Inertsil ODS-SP C18 column (5 $\mathrm{m}, 4.6 \times 250 \mathrm{~mm}$; GL Sciences, Tokyo, Japan) was used. The column was maintained at $30{ }^{\circ} \mathrm{C}$ and the absorbance detector wavelength was $363 \mathrm{~nm}$. The chromatographic separation was performed on isocratic elution with acetonitrile- $0.1 \%$ formic acid $(80: 20, v / v)$ and acetonitrile- $0.1 \%$ formic acid $(45: 55, v / v)$ for $\mathrm{G} 2$ and CPT, respectively. The flow velocity was $1.0 \mathrm{~mL} / \mathrm{min}$. The injection volume of the supernatant samples was $20 \mu \mathrm{L}$.

To determine the concentration of G2 and CPT in the stability investigation, a C18 $(5 \mu \mathrm{m}$, $4.6 \times 250 \mathrm{~mm}$, Elite, Dalian, China) reverse-phase column was used. The chromatographic separation was performed on isocratic elution with acetonitrile- $0.1 \%$ formic acid $(80: 20, v / v)$ and acetonitrile- $0.1 \%$ formic acid $(60: 40, v / v)$ for G2 and CPT, respectively. Other conditions were the same as cell model.

To determine G2 and CPT concentration in plasma and tissue homogenate, an Inertsil ODS-SP C18 column $(5 \mu \mathrm{m}, 4.6 \times 250 \mathrm{~mm}$; GL Sciences) was used. The mobile phase was made up of acetonitrile (A) and 0.1\% formic acid (B). CPT was separated by an isocratic elution with 35:65 (v/v) A:B. A gradient eluant ((A:B) 0-3 min: 35:65; 3-8 min: from 35:65 to 80:20; 8-20 min: 80:20; 20-21 min: from 80:20 to 35:65; 21-25 min: 35:65) was used to separate G2. Other conditions were the same as cell model.

\subsection{Method Validation}

As G2 and CPT were studied in vivo, the analytical method was validated. Method validation was conducted according to the acceptance criteria established by U.S. Food and Drug Administration for bioanalytical method validation. The validation parameters included specificity, linearity, accuracy, precision, recovery, matrix effect and stability.

The selectivity of the method was assessed by comparing the chromatograms of six different sources of blank samples with the corresponding spiked samples. The calibration curves were constructed by plotting the peak area ratio of G2 or CPT to IS (y) versus the concentration ( $\mathrm{x}$, range from 0.015 to $2.4 \mu \mathrm{M}$ ). The regression equations were obtained using least squares linear regression weighted by $1 / x^{2}$. The inter-day and intra-day accuracy and precision were evaluated using three concentrations $(0.03,0.3,1.2 \mu \mathrm{M})$ of QC samples on the same day and different validation days, respectively. The accuracy was represented by the percentage (\%) of mean measured concentration to spiked concentration. Precision was represented by relative standard deviation (RSD). Extraction recovery was calculated by dividing the peak areas of QC samples at three concentrations with the unextracted standards. The stability was evaluated using QC samples at three concentrations to confirm freeze-thaw stability (three cycles), long-term freeze stability ( $-80^{\circ} \mathrm{C}$ for four weeks), short-term stability (room temperature for 4-24 h). The results of stability were expressed as the percentage (\%) of mean measured concentration in this experiment to the initial concentration. The matrix effect was 
obtained via comparing the peak areas of spiked solution of post-extracted blank plasma with pure standard solution.

\subsection{Data Analysis}

The pharmacokinetic analysis using a non-compartmental model with the PKSolver pharmacokinetic software (China Pharmaceutical University, Nanjing, China) was conducted to calculate the key parameters of G2 and CPT. In order to evaluate whether the conjugate had the trend of liver targeting, liver targeting index (LTI) was calculated by Equation (1) below:

$$
\mathrm{LTI}=\mathrm{AUC}_{0-\mathrm{t}^{\prime}} \text { in liver } / \mathrm{AUC}_{0-\mathrm{t}} \text { in plasma }
$$

where $\mathrm{AUC}_{0-\mathrm{t}^{\prime}}$ in liver is the $\mathrm{AUC}_{0-\mathrm{t}}$ of drug in liver and $\mathrm{AUC}_{0-\mathrm{t}}$ in plasma is the $\mathrm{AUC}_{0-\mathrm{t}}$ of drug in plasma. All the data were presented as mean $\pm \mathrm{SD}$. Statistical analysis were performed by Student's $t$-test using the SPSS 22.0 statistical software program (IBM company, Denver, CO, USA), and $p<0.05$ was used as the criterion for statistical significant.

\section{Conclusions}

In this interesting study, we evaluated the potential liver targeting and stability of deoxycholic acid-CPT conjugate $\mathbf{G} 2$ in vitro as well as its potential liver targeting in vivo. The results of competitive inhibition of antitumor activitiy against HepG2 cells suggested that G2 could be taken up by bile acid transporters for its conjugation with deoxycholic acid. Subsequent investigation of uptake into HepG2 cells in 2D cell model and 3D model further demonstrated that conjugation with deoxycholic acid changed the transport pathway of CPT and the uptake mediated by bile acid transporters played an important role in the cellular internalization of G2 in HepG2 cells. Stability tests in SGF and SIF as well as rat plasma showed that conjugation with deoxycholic acid significantly increased the stability in vitro. Body distribution study in mice demonstrated that G2 increased the LTI by 1.67-fold compared with CPT. In short, the current study indicates that coupling with deoxycholic acid moiety is a feasible means to enhance the potential liver targeting and the stability of CPT, which provide a theoretical basis for preclinical trials of G2.

Supplementary Materials: Tables S1-S4 are available on line. Table S1: Linearity of G2 and CPT in biological samples $(\mathrm{n}=3)$. Table S2: Accuracy and precision data for G2 and CPT in biological samples $(\mathrm{n}=6)$. Table S3: Recovery and matrix effect of G2 and CPT in biological samples $(n=6)$. Table S4: Stability data for G2 and CPT in biological samples $(n=6)$.

Author Contributions: Data Curation, L.X. and E.Y.; Writing-Original Draft Preparation, L.X.; Writing-Review\& Editing, Q.L. and H.Y.; Project Administration, L.X. and Q.L.; Funding Acquisition, Q.L.

Funding: This work was financially supported by Qianjiang talents project in Zhejiang province.

Conflicts of Interest: The authors declare no conflict of interest, financial or otherwise.

\section{References}

1. Wani, M.C.; Wall, M.E.; Cook, C.E.; Palmer, K.H.; McPhail, A.T.; Sim, G.A. Plant antitumor agents. I. The isolation and structure of camptothecin a novel alkaloidal leukemia and tumor inhibitor from Camptotheca acuminata. J. Am. Chem. Soc. 1966, 88, 3888-3890.

2. Hertzberg, R.P.; Caranfa, M.J.; Hecht, S.M. On the mechanism of topoisomerase I inhibition by camptothecin: Evidence for binding to an enzyme-DNA complex. Biochemistry 1989, 28, 4629-4638. [CrossRef]

3. Kehrer, D.F.; Soepenberg, O.; Loos, W.J.; Verweij, J.; Sparreboom, A. Modulation of camptothecin analogs in the treatment of cancer: A review. Anti-Cancer Drug 2001, 12, 89-105. [CrossRef]

4. Park, E.S.; Kang, S.; Yoo, K.; Lee, M.Y.; Yoo, H.S.; Hong, J.T.; Shin, H.S.; Kim, B.; Yun, Y.P. Camptothecin inhibits platelet-derived growth factor-BB-induced proliferation of rat aortic vascular smooth muscle cells through inhibition of PI3K/Akt signaling pathway. Exp. Cell Res. 2013, 319, 982-991. [CrossRef] [PubMed] 
5. $\quad$ Liu, Y.Q.; Li, W.Q.; Morris-Natschke, S.L.; Qian, K.; Yang, L.; Zhu, G.X.; Wu, X.B.; Chen, A.L.; Zhang, S.Y.; Nan, X.; et al. Perspectives on biologically active camptothecin derivatives. Med. Res. Rev. 2015, 35, 753-789. [CrossRef] [PubMed]

6. Hsu, J.L.; Ho, Y.F.; Li, T.K.; Chen, C.S.; Hsu, L.C.; Guh, J.H. Rottlerin potentiates camptothecin-induced cytotoxicity in human hormone refractory prostate cancers through increased formation and stabilization of topoisomerase I-DNA cleavage complexes in a PKC $\delta$-independent pathway. Biochem. Pharmacol. 2012, 84, 59-67. [CrossRef]

7. Yeo, C.D.; Lee, S.H.; Kim, J.S.; Kim, S.J.; Kim, S.C.; Kim, Y.K.; Kang, H.H.; Yoon, H.K.; Song, J.S.; Moon, H.S.; et al. A multicenter phase II study of belotecan, a new camptothecin analogue, in elderly patients with previously untreated, extensive-stage small cell lung cancer. Cancer Chemoth. Pharm. 2013, 72, 809-814. [CrossRef]

8. Li, Q.Y.; Zu, Y.G.; Shi, R.Z.; Yao, L.P. Review camptothecin: Current perspectives. Curr. Med. Chem. 2006, 13, 2021-2039. [CrossRef] [PubMed]

9. He, X.G.; Lu, W.; Jiang, X.Q.; Cai, J.C.; Zhang, X.W.; Ding, J. Synthesis and biological evaluation of bis and monocarbonate prodrugs of 10-hydroxycamptothecins. Bioorgan. Med. Chem. 2004, 12, 4003-4008. [CrossRef]

10. Oledzka, E.; Horeglad, P.; Gruszczynska, Z.; Plichta, A.; Nalecz-Jawecki, G.; Sobczak, M. Polylactide conjugates of camptothecin with different drug release abilities. Molecules 2014, 19, 19460-19470. [CrossRef] [PubMed]

11. Li, Q.Y.; Zhu, Q.C.; Zhang, X.K.; Hua, C.L.; Deng, X.Q.; Zhao, T.F.; Sun, B.H. A liquid chromatography-tandem mass spectrometry method for pharmacokinetics and tissue distribution of a camptothecin quaternary derivative in rats. Fitoterapia 2013, 90, 57-64. [CrossRef]

12. Du, H.Z.; Huang, Y.; Hou, X.Y.; Quan, X.P.; Jiang, J.W.; Wei, X.H.; Liu, Y.; Li, H.Y.; Wang, P.H.; Zhan, M.X.; et al. Two novel camptothecin derivatives inhibit colorectal cancer proliferation via induction of cell cycle arrest and apoptosis in vitro and in vivo. Eur. J. Pharm. Sci. 2018, 123, 546-559. [CrossRef]

13. Guo, Z.P.; Zhou, X.Z.; Xu, M.Z.; Tian, H.Y.; Chen, X.S.; Chen, M.W. Dimeric camptothecin-loaded RGD-modified targeted cationic polypeptide-based micelles with high drug loading capacity and redox-responsive drug release capability. Biomater. Sci. 2017, 5, 2501-2510. [CrossRef] [PubMed]

14. Zhang, Z.; Cai, H.X.; Liu, Z.J.; Yao, P. Effective enhancement of hypoglycemic effect of insulin by liver-targeted nanoparticles containing cholic acid-modified chitosan derivative. Mol. Pharm. 2016, 13, 2433-2442. [CrossRef]

15. Chen, D.Q.; Wang, X.; Chen, L.; He, J.X.; Miao, Z.H.; Shen, J.K. Novel liver-specific cholic acid-cytarabine conjugates with potent antitumor activities: Synthesis and biological characterization. Acta Pharmacol. Sin. 2011, 32, 664-672. [CrossRef]

16. Dong, Z.Q.; Li, Q.; Guo, D.; Shu, Y.; Polli, J.E. Synthesis and evaluation of bile acid-ribavirin conjugates as prodrugs to target the liver. J. Pharm. Sci.-US 2015, 104, 2864-2876. [CrossRef] [PubMed]

17. Li, Y.; Zhu, C.Y. Enhanced hepatic-targeted delivery via oral administration using nanoliposomes functionalized with a novel DSPE-PEG-cholic acid conjugate. RSC Adv. 2016, 6, 28110-28120. [CrossRef]

18. Zhang, D.; Li, D.P.; Shang, L.; He, Z.G.; Sun, J. Transporter-targeted cholic acid-cytarabine conjugates for improved oral absorption. Int. J. Pharmaceut. 2016, 511, 161-169. [CrossRef]

19. Vivian, D.; Polli, J.E. Synthesis and in vitro evaluation of bile acid prodrugs of floxuridine to target the liver. Int. J. Pharmaceut. 2014, 475, 597-604. [CrossRef]

20. Halilbasic, E.; Claudel, T.; Trauner, M. Bile acid transporters and regulatory nuclear receptors in the liver and beyond. J. Hepatol. 2013, 58, 155-168. [CrossRef]

21. Dawson, P.A. Role of the intestinal bile acid transporters in bile acid and drug disposition. Handb. Exp. Pharmacol. 2011, 201, 169-203.

22. Li, Q.Y.; Gao, Y.; Qiu, W.; Zu, Y.G.; Su, L.; He, W.N.; Deng, X.Q. Synthesis and anti-tumour activity of novel camptothecin-bile acid analogues. Lett. Drug Des. Discov. 2011, 8, 698-703. [CrossRef]

23. Li, X.N.; Zhao, T.F.; Cheng, D.P.; Chu, C.; Tong, S.Q.; Yan, J.Z.; Li, Q.Y. Synthesis and biological activity of some bile acid-based camptothecin analogues. Molecules 2014, 19, 3761-3776. [CrossRef]

24. Jarockyte, G.; Dapkute, D.; Karabanovas, V.; Daugmaudis, J.V.; Ivanauskas, F.; Rotomskis, R. 3D cellular spheroids as tools for understanding carboxylated quantum dot behavior in tumors. Biochim. Biophys. Acta (BBA) Gen. Subj. 2018, 1862, 914-923. [CrossRef] 
25. Sarisozen, C.; Abouzeid, A.H.; Torchilin, V.P. The effect of co-delivery of paclitaxel and curcumin by transferrin-targeted PEG-PE-based mixed micelles on resistant ovarian cancer in 3-D spheroids and in vivo tumors. Eur. J. Pharm. Biopharm. 2014, 88, 539-550. [CrossRef]

26. Van den Brand, D.; Veelken, C.; Massuger, L.; Brock, R. Penetration in 3D tumor spheroids and explants: Adding a further dimension to the structure-activity relationship of cell-penetrating peptides. Biochim. Biophys. Acta (BBA) Biomembr. 2018, 1860, 1342-1349. [CrossRef]

27. Li, D.Z.; Li, Y.; Chen, X.G.; Zhu, C.G.; Yang, J.; Liu, H.Y.; Pan, X.D. Synthesis and antitumor activity of heterocyclic acid ester derivatives of 20S-camptothecins. Chin. Chem. Lett. 2007, 18, 1335-1338. [CrossRef]

28. Chu, C.; Xu, J.L.; Cheng, D.P.; Li, X.N.; Tong, S.Q.; Yan, J.Z.; Li, Q.Y. Anti-proliferative and apoptosis-inducing effects of camptothecin-20(s)-O-(2-pyrazolyl-1) acetic ester in human breast tumor MCF-7 cells. Molecules 2014, 19, 4941-4955. [CrossRef]

29. Zhao, J.C.; Lu, H.X.; Yao, Y.; Ganda, S.; Stenzel, M.H. Length vs. stiffness: Which plays a dominant role in the cellular uptake of fructose-based rod-like micelles by breast cancer cells in 2D and 3D cell culture models? J. Mater. Chem. B 2018, 6, 4223-4231. [CrossRef]

30. Zeng, Z.; Shen, Z.L.; Zhai, S.; Xu, J.L.; Liang, H.; Li, Q.Y. Transport of curcumin derivatives in Caco-2 cell monolayers. Eur. J. Pharm. Biopharm. 2017, 117, 123-131. [CrossRef]

31. Yang, T.C.; Chao, H.F.; Shi, L.S.; Chang, T.C.; Lin, H.C.; Chang, W.L. Alkaloids from Coptis chinensis root promote glucose uptake in C2C12 myotubes. Fitoterapia 2014, 93, 239-244. [CrossRef]

32. He, C.B.; Yin, L.C.; Tang, C.; Yin, C.H. Size-dependent absorption mechanism of polymeric nanoparticles for oral delivery of protein drugs. Biomaterials 2012, 33, 8569-8578. [CrossRef]

33. Yin, L.C.; Ding, J.Y.; He, C.B.; Cui, L.M.; Tang, C.; Yin, C.H. Drug permeability and mucoadhesion properties of thiolated trimethyl chitosan nanoparticles in oral insulin delivery. Biomaterials 2009, 30, 5691-5700. [CrossRef]

34. Park, S.J.; Garcia, C.V.; Shin, G.H.; Kim, J.T. Development of nanostructured lipid carriers for the encapsulation and controlled release of vitamin D3. Food Chem. 2017, 225, 213-219. [CrossRef]

Sample Availability: Samples of the compounds are not available from the authors.

(C) 2019 by the authors. Licensee MDPI, Basel, Switzerland. This article is an open access article distributed under the terms and conditions of the Creative Commons Attribution (CC BY) license (http://creativecommons.org/licenses/by/4.0/). 